\title{
Molecular dynamics simulations of molecular sliders on biopolymers
}

Yanxiao Han ${ }^{1}$, Petr Král ${ }^{1,2,3}$

${ }^{1}$ Department of Chemistry, ${ }^{2}$ Physics and ${ }^{3}$ Biopharmaceutical Sciences, University of Illinois at Chicago, USA.

\section{Introduction}

Inspired by the efficient sliding and hopping motion of proteins along polynucleotides, we study the diffusive transport and binding of molecular sliders on polymeric track which does not require a chemical fuel.
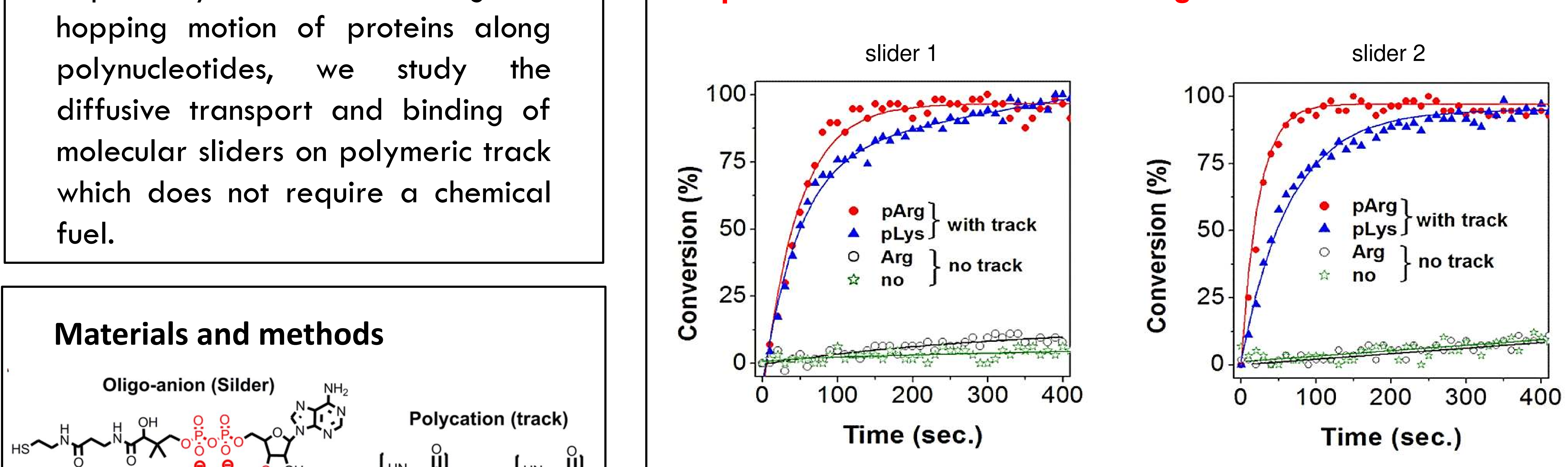

Slider 1 is in a $25 \mathrm{mM}$ MES solution and slider 2 is in a $22 \mathrm{mM}$ MOPS solution. In the presence of molecular tracks, the sliders react about 100 times faster.

MD simulations of sliders on a poly-arginine track

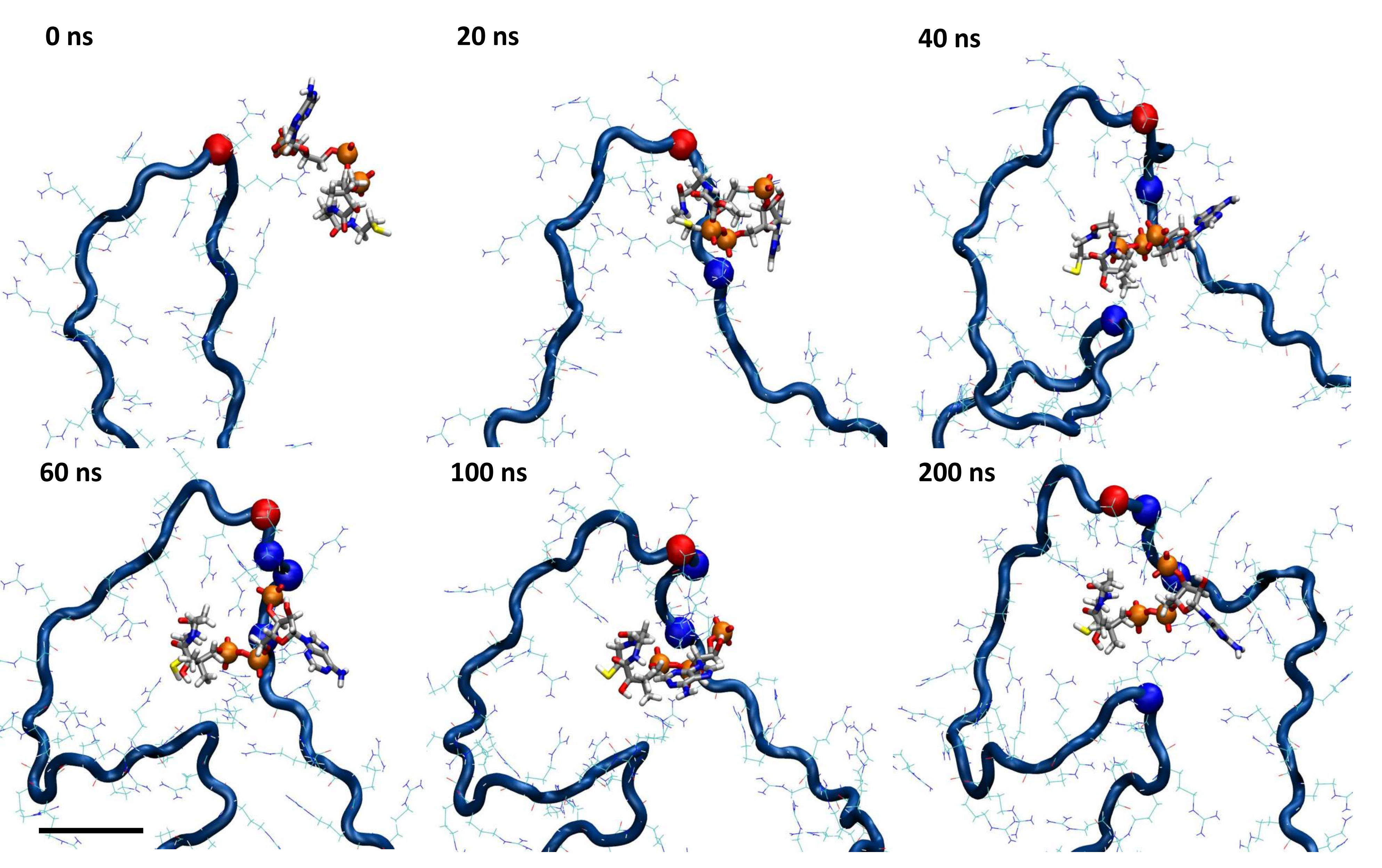

Slider 1 on pArg, the pArg track is relatively curled and forms many transient loops. Oppositely charged groups hold sliders on track during their $1 \mathrm{D}$ diffusive motion.

P. Král, and W. T. Shao, Y. Han, H. Qian, L. Vuković, J,Mech

Simulations were performed by Yanxiao Han under the advice of Prof. Email : yhan57@uic.edu
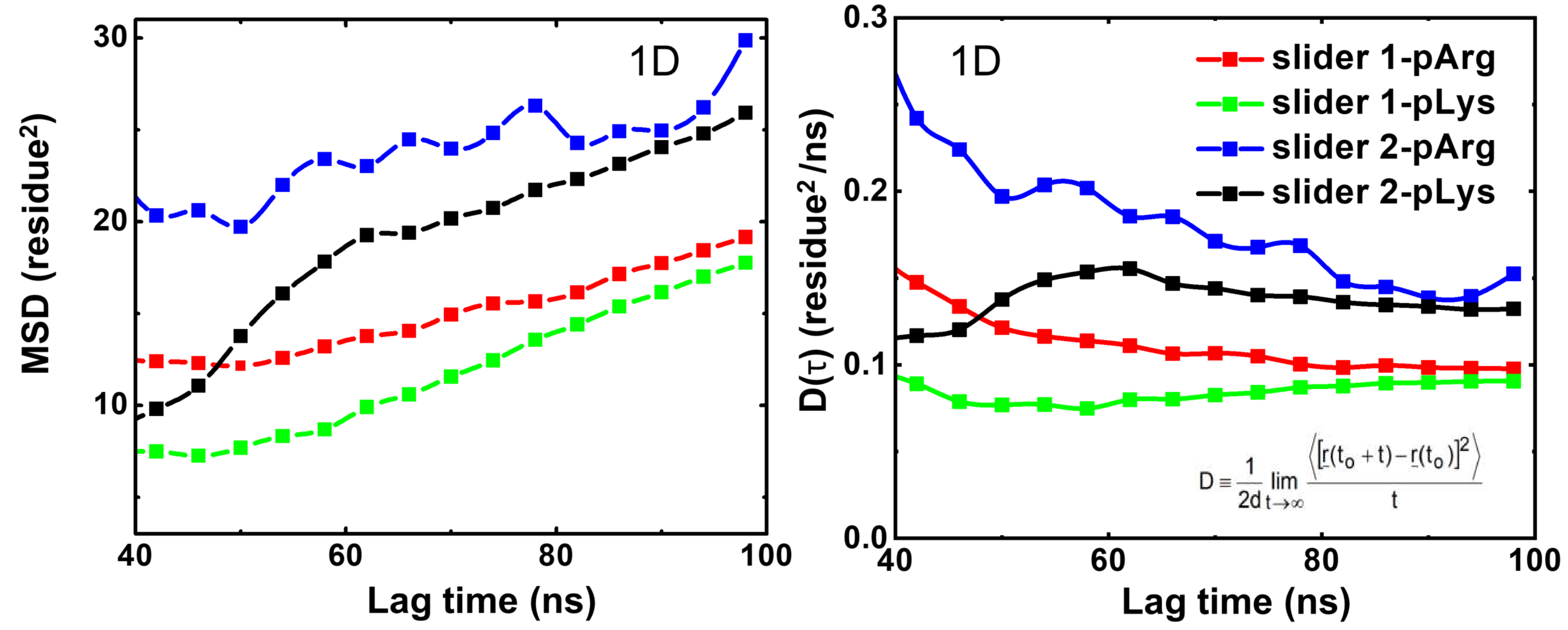

(Left) Sliders move 4-5 residues along tracks within $100 \mathrm{~ns}$.

(Right) The 1D diffusion constants (on the chain - not 3D) are larger on PARG than on pLYS, which is consistent with an enhancement of the experimental reaction rates:

Slider 1 -pARG (enhanced by 113 -fold) $>$ Slider 1-pLYS $(110)$ Slider2-pARG (149) > Slider2-pLYS (77).
Free energy profile

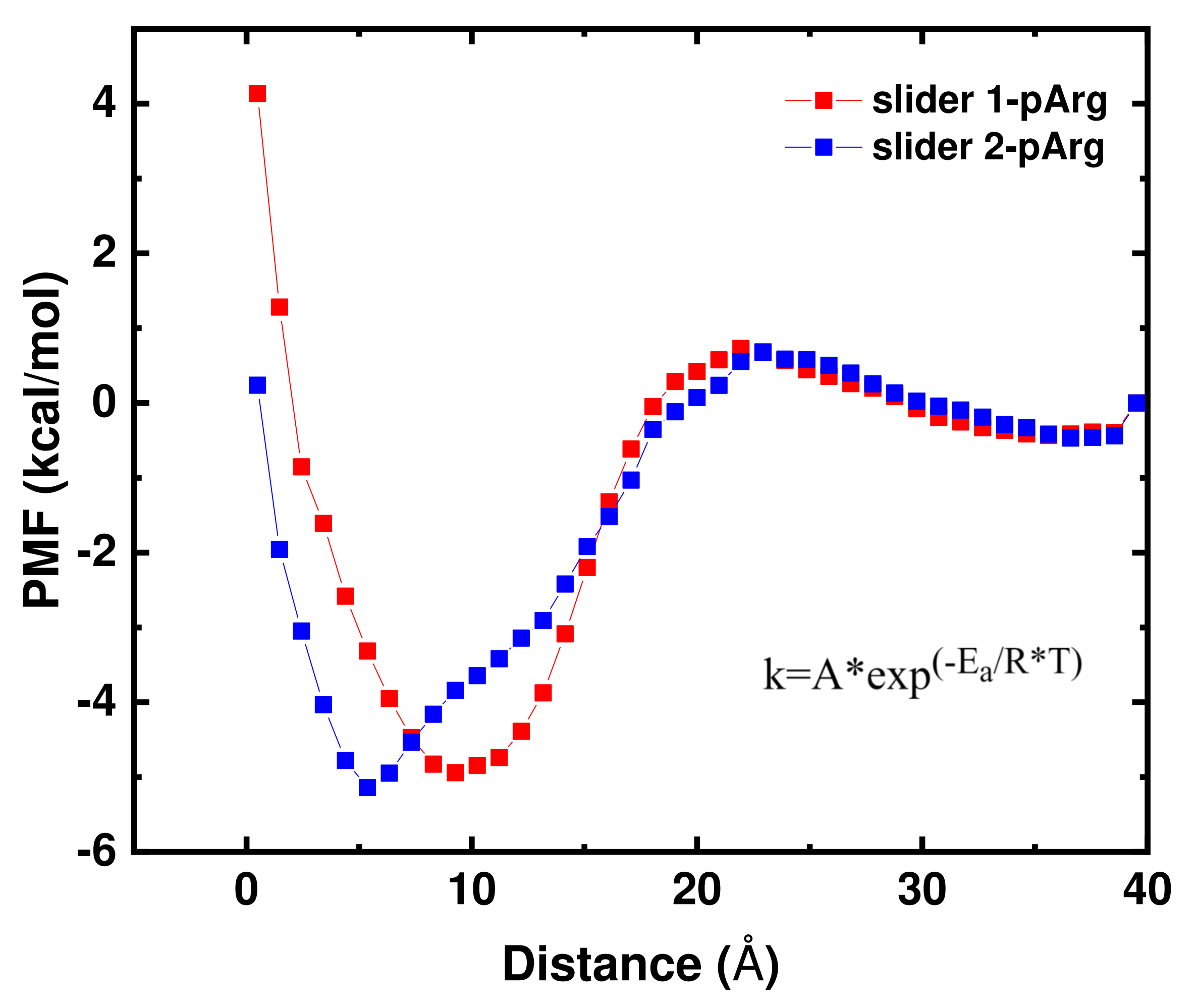

Slider $1 / 2$ on $\mathrm{pArg}$
Sliders can hop on and between tracks
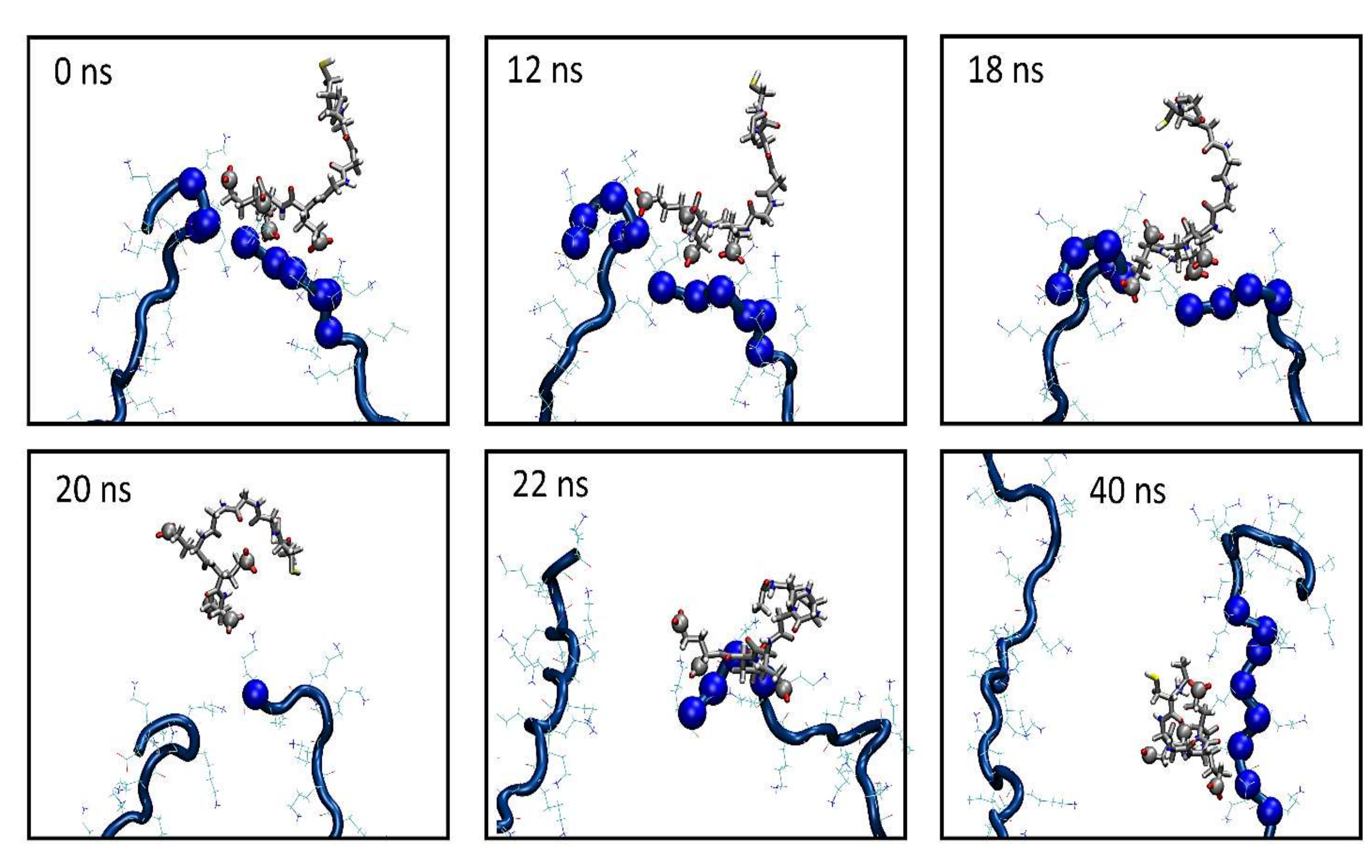

Slider2 on short plys
Experiments were done in Prof. Huck's lab.
Slider $1(2)$ unbinding rate is $\mathrm{k}=1.54 \times 106 \mathrm{~s}-1$ and the binding time is $\sim 0.6(0.7) \mu \mathrm{s}$ (Arrhenius frequency factor $2.5 \times 10^{10} \mathrm{~s}^{-1}$ ) 